\title{
Two- and Three-Dimensional Numerical Experiments Representing Two Limiting Cases of an In-Line Pair of Finger Seal Components
}

\author{
M. J. Braun \\ Department of Mechanical Engineering, University of Akron, Akron, Ohio, USA
}

V. V. Kudriavtsev

CFD Canada, Toronto, Ontario, Canada

B. M. Steinetz and M. P. Proctor

NASA Glenn Research Center, Cleveland, Ohio, USA

The work presented here concerns the numerical development and simulation of the flow, pressure patterns, and motion of a pair of fingers arranged one behind the other and axially aligned in line. The fingers represent the basic elemental component of a finger seal and form a tight seal around the rotor. Yet their flexibility allows compliance with rotor motion and, in a passive-adaptive mode, compliance with the hydrodynamic forces induced by the flowing fluid. Although this article does not treat the actual staggered configuration of a finger seal, the in-line arrangement represents a first step toward that final goal. The numerical two-dimensional (axial-radial) and three-dimensional results presented herein were obtained using a commercial package (CFD-ACE + ). Both models use an integrated numerical approach, which couples the hydrodynamic fluid model based on Navier-Stokes equations to the solid mechanics code that models the compliance of the fingers.

Keywords Finger-seal, Numerical simulation, Two-dimensional, Three-dimensional

For small-diameter turbines (with short blades), wear of the blade tip is particularly troublesome because it generates a much

Received 5 July 2002; accepted 5 July 2002.

Address correspondence to M. J. Braun, Department of Mechanical Engineering, University of Akron, Akron, OH 44352, USA. E-mail: mjbraun@uakron.edu larger percentage of leakage flow in comparison to larger diameter turbines with similar wear. The basic causes of clearance increase reside with the radial displacements of the rotor which, in time, create clearances that are generally larger than those desirable for efficient operation. These displacements can be caused by (1) differential tandem thermal responses of the case and the rotor, caused by operational transients; (2) ovalization due to nonaxisymmetrical temperatures and/or loads; (3) centrifugal and gyroscopic loads; and (4) thrust, maneuver, or air turbulence loads. The real bottom line of the effect of seal leakages can be seen in the change in the specific fuel consumption (SFC), engine effectiveness, and overhaul times. The leakage causes enumerated above are not necessarily specific to the turbine, but they also apply to the high-pressure and low-pressure compressors, even though the thermal environment is generally less hostile. According to Ludwig (1978), the SFC can increase (due to leakage) by as much as $0.51 \%$ in a high-pressure compressor, $0.58 \%$ in a high-pressure turbine (HPT), and $0.69 \%$ in a low-pressure turbine. The compliant shroud/seal (whether outfitted with brush or finger seals) appears to be an ideal candidate for the role of passive-adaptive mitigation of this type of leakage. It provides a compliant buffer for the HPT blades and due to its compliance will keep, over time, the operating clearances significantly smaller than the currently used rigid shrouds. It should be noted that the technology of compliant shaft seals was revolutionized about 15 years ago, when brush seals were proposed to replace the labyrinth seals at many locations along the compressor's and turbine's shafts. Today, brush seals are in operation as shaft seals in Rolls-Royce, Pratt and Whitney, and GE jet engines. 

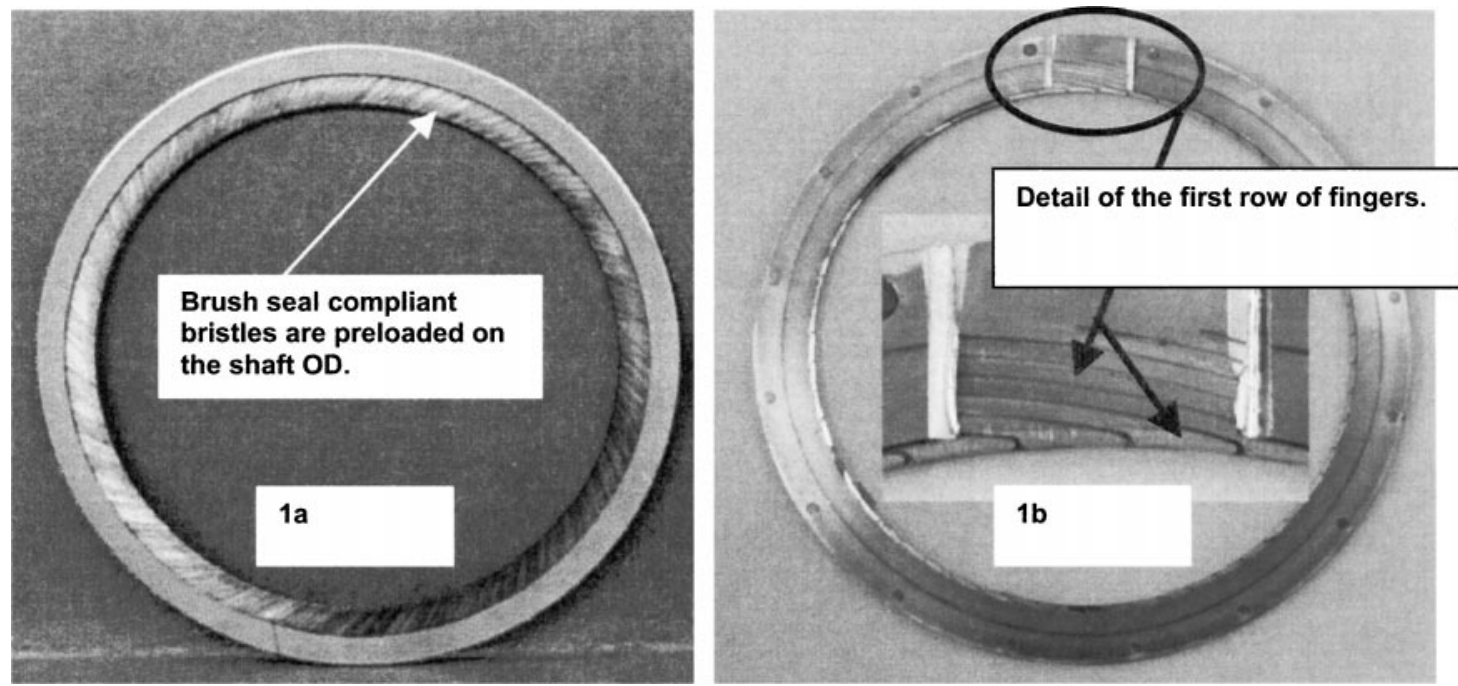

FIGURE 1

Typical brush and finger seals.

\section{BRIEF REVIEW OF THE STATE OF THE ART OF COMPLIANT SEALS}

\section{Brush Seals}

Sealing at the brush-seal/shaft interface is ensured by tight packing of the bristles and interference tolerance fit between the seal's inner diameter and shaft's outer diameter (Fig. 1). Leakage occurs both at the brush/rotor interface and through the brush's bristle pack. It is now universally recognized that this embodiment is much more effective than the labyrinth seal, and its deterioration is much delayed. There has been a considerable amount of experimental work that has advanced the state of the art of these types of seals (Atkinson and Bristol, 1992; Chupp and Dowler, 1991a; Ferguson, 1988; Forry, 1993; Hendricks et al., 1992; Proctor et al., 1996). During the same period, an effort was also afoot to validate numerical simulations through comparisons to the available experimental data. Thus, early on, Braun and colleagues (1990) and Hendricks and colleagues (1991) offered a bulk flow model for flow through the body of a brush, based on analytical and experimental developments concerning flow in porous media. The analytical results, when compared with experimental data, showed relatively good coincidence. The same year, Mullen and colleagues (1990) presented numerical results based on a finite element model that simulated the flow field of a $6 \times 6$ uniformly spaced bristle matrix. The maximum differential between the experimental and numerical results was observed at low flows and was approximately $30 \%$. Chupp and Holle $(1991,1994)$ also offered alternate bulk flow models for leakage through a randomly distributed bristle bed.

\section{Finger Seals}

A finger seal (Fig. 1b) also represents a compliant seal configuration (Fig. 2). Earlier versions of the finger seal were pi- oneered by Heydrich (1991), Johnson and colleagues (1992), and Mackay and colleagues (1991a, 1991b). What differentiates finger seals and makes them preferable to brush seals is their potential hydrodynamic lifting capabilities and, thus, their noncontacting nature. The fingers' compliance allows both axial and radial adjustment to rotor excursions without damage to the integrity of the seal. Their potential lifting capability eliminates the wear factor, thus considerably increasing their life span over that of rigid seals. The fundamental geometry of a finger seal can be seen in Figures $1 \mathrm{~b}$ and 2. The shape of the finger (stick plus pad) is etched chemically into the material by means of a photo

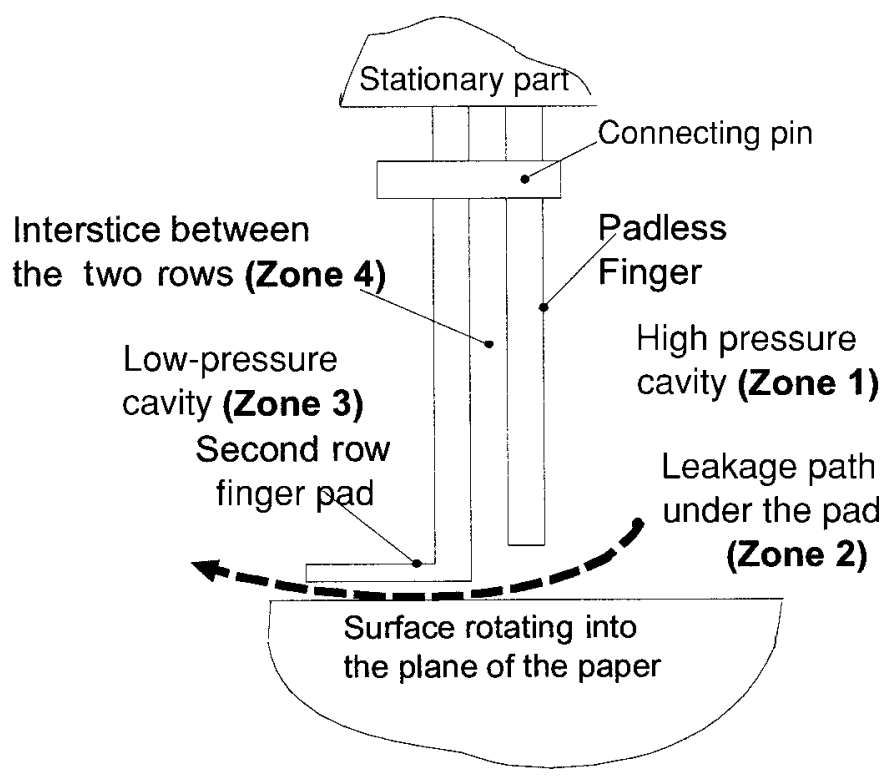

FIGURE 2

Two-row configuration of a finger seal, with finger pad on the high-pressure side, longitudinal cross-section. 
process that allows the cutting of the thin interstices separating the individual fingers. The stick ends with an enlarged formation that has a geometry similar to that of the pad shown in Figure 2. The root of the stick joins the annulus shown in Figure 1b, from which the whole finger seal is cut. This configuration allows the finger to move freely, like a cantilever beam in its space, and thus exhibit compliance with the external forces acting on it. To form a full seal, two or more annular laminates are assembled axially in a staggered arrangement such that fingers from the upstream laminates cover the interstices between the fingers of the downstream laminates (Fig. 1b). In general, the width of the seal is such that in a turbine blade tip application it covers the entire axial width of the turbine blade; in a shaft seal, the width is chosen with fewer restrictions. In the latter embodiment, the shaft seals, have already been successfully tested as interstage and buffer seals at the NASA Glenn Research Center. A report by Hendricks and colleagues (1994) reviews four compliant seal concepts, including finger seals that have the potential to be noncontacting seals. The authors provide a comparative table of experimental results that show the performance of contacting finger seals to be better in terms of leakage than both the labyrinth and the brush seals. They also offer a bulk model analysis similar to the one performed by Braun and colleagues (1990) and Hendricks and colleagues (1991) for the brush seal. Steinetz and colleagues (1998) also mention the finger seal as a potential film-riding, noncontacting candidate for both shaft and blade tip seals. Finally, Arora and colleagues (1999) present a detailed evaluation of a low-hysteresis finger seal, finding that the hysteresis and endurance performance were encouraging and that typical leakage is 20 to $70 \%$ less than that of a four-knife labyrinth seal. The seal was evaluated at speeds as high as $945 \mathrm{ft} / \mathrm{sec}(288 \mathrm{~m} / \mathrm{sec})$ and at an $80 \mathrm{psi}(552 \mathrm{kPa})$ pressure differential.

\section{SCOPE OF WORK}

Just like the brush seals, the finger seals are mounted on the rotor with a certain interference fit. Finger seals exhibit two advantages over brush seals: (1) the manufacturing costs are much lower than those of brush seals; and (2) the finger pad geometry offers the potential for pad lift off. The purpose of this article is to present, for the first time, distributed models in two and three dimensions. We analyze an assembly of two fingers arranged in line. The two-dimensional model considers flow only in the axial and radial directions (Fig. 2) with no leakage between adjacent fingers. The three-dimensional model also considers the flow in the circumferential direction (Figs. 3 and 4). The fingers have freedom of motion in all directions. Both models incorporate fluid-structure interaction in order to account for the fingers' compliance. The two-dimensional approach, in fact, represents the limiting best-case scenario, when leakage occurs only through the area of the gap under the finger pad. The three-dimensional model represents the limiting worst-case scenario, when flow leakage moves through the pad/shaft gap and in an unrestrained mode inbetween the fingers. Both the twodimensional and the three-dimensional models combine in an integrated mode the Navier-Stokes equations with an elasticity model and shaft rotation, thus solving jointly for seal leakage and elastic deformation of the finger pad. The basic goal of this preliminary work is to establish limit cases and to gain insight into the physics of flow and finger motion. Eventually, this work will deliver a tool for parametric evaluation of finger seal designs that ensure both the lifting of the finger and acceptable leakages.

\section{GEOMETRY OF THE FINGERS}

We present here a detailed layout of the three-dimensional computational domain and its construction, with only the

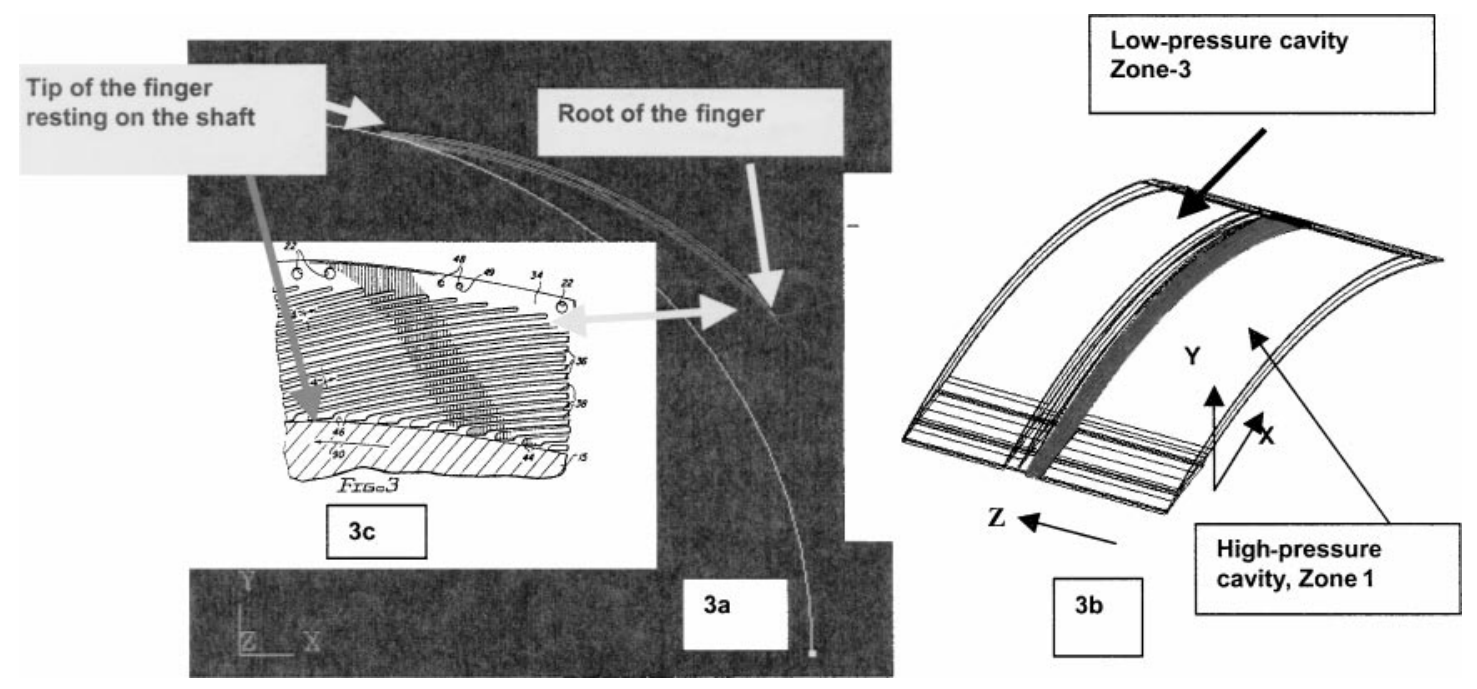

FIGURE 3

(a) Typical position of one finger, with its root and its pad. (b) Arrangement of fingers inbetween the cavities. Frontal view of a wafer containing padless fingers (From Arora, 1998). 


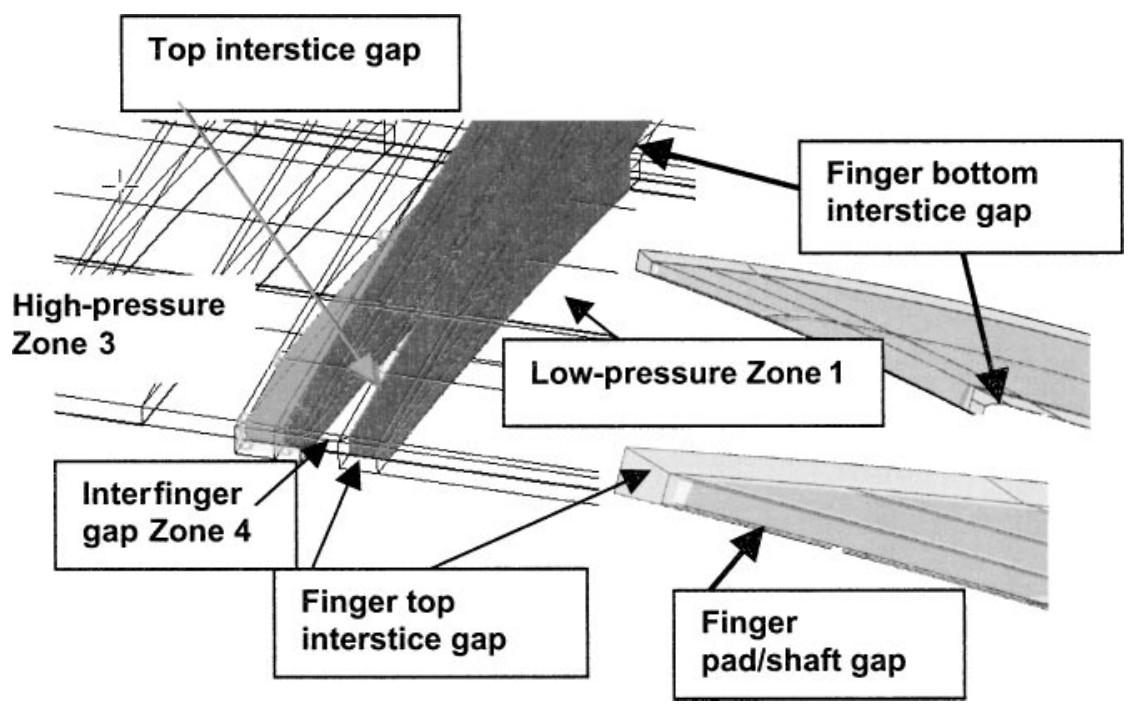

FIGURE 4

Details of the computational domains around the pair of in-line fingers.

mention that the grid used for the two-dimensional simulation was obtained by eliminating the circumferential coordinate from the three-dimensional simulation. The basic geometry of the fingers assembly studied here is presented in the radial-axial crosssection in Figures 3 and 4. This is a modification of the Arora (1998) patent in that the finger on the high-pressure side does not incorporate a pad but ends about 0.015 in above the surface of the rotating shaft. The elimination of this pad helps in the overall hydrodynamic lifting process of the pad finger off the shaft. The three-dimensional grid (incorporating the high-pressure cavity (Zone 1); the fingers; the interstice Zone 4 between the fingers; the top and bottom gaps surrounding the stick and pad; and the low-pressure Zone 3) is shown in Figures $3 a, 3 b$, and 4 . The shaft-to-pad clearance is 0.0005 in $(0.0127 \mathrm{~mm})$, and the elongated interstitial top and bottom gaps that separate upstream and downstream sequential fingers are 0.015 in $(0.381 \mathrm{~mm})$. These interstice gaps have a geometry that is controlled by a circumferential logarithmic curvature. The full-seal geometry has 72 fingers per row and two to three rows in the axial direction, but our computational setup was limited to a pair of upstream and downstream in-line fingers (as discussed) and the gaps surrounding them. The third (axial) dimension shows the cavity in front, the cavity inbetween, and the cavity behind the two axially neighboring fingers (Fig. 3b). The low-pressure finger pad, if properly designed, should be able to generate sufficient hydrodynamic lifting force to offset the static pressure applied on the top of the pad by the environment. The pad thus lifted generates enough pressure drop in the axial direction to act as a seal and to glide in a compliant fashion over the shaft.

\section{Gridding}

The three-dimensional grid was obtained by extrusion of the two-dimensional $\mathrm{X}$-Y grid into the third $(\mathrm{Z})$ dimension, as shown in Figure 3b. This direction was aligned with the axis of the shaft. The first two-dimensional X-Y plane boundary perpendicular on the longitudinal axis was created at the position where the highpressure cavity was starting (the overall domain inlet; Fig. 3b). The two fingers and domain boundaries were projected on this plane. Grid distributions were then assigned to all of these geometric formations. The overall three-dimensional computational model incorporated 403,696 cells in 152 subdomains. The grid used 60 nodes in the $\mathrm{X}$ direction, 85 nodes in the $\mathrm{Y}$ direction, and 100 nodes in the $\mathrm{Z}$ direction. The large memory requirements were the result of a significant amount of three-dimensional prismatic finite element analysis (fea) nodes. The fea portion of the mesh was used for the solid finger model and had to match the fluid mesh at their interface. The fluid mesh required very small grid sizes in certain small footprint areas, causing computational overhead for the fea grid. The fluid-structure formulation required matching grid interfaces, and the fluid cells had to be structured/prismatic to allow the transfinite interpolation necessary to account for the grid movement.

\section{NUMERICAL MODEL AND SOLUTION}

\section{Computational Model}

The computational engine, $\mathrm{CFD}-\mathrm{ACE}+$, is a commercial software package available from CFDRC (Huntsville, Alabama). The algorithm solves the full Navier-Stokes equations in a Cartesian two-dimensional axisymmetrical system of coordinates, with rotating boundary conditions at the shaft surface. We utilized a conjugate gradient algebraic solver with preconditioning. The flow problem with no finger deformation typically converged in 300 iterations. The flow problem with finger deformation utilized the transient solution method. The computation used five iterations with very large time steps and allowed 
100 subiterations on each time step. The algorithm utilized first-order approximations for upwind terms and second-order finite elements. Prismatic grids were used for both flow and solid domains. CFD-ACE + uses absolute convergence criteria, which for pressure fields usually requires convergence to four orders of magnitude. Convergence for finger deflections is monitored on each time step, and usually after five transient iterations, the solution converges. The code uses an add-on module, FEMSTRESS, that interacts in a feedback mode with CFD$\mathrm{ACE}+$ to enable the calculation of stresses and deformations that develop in the finger due to the pressures and shear associated with the fluid motion. In our study we utilized FEMSTRESS's two-way transient coupling to accommodate grid motion due to the deformation of solid fingers. Solid grid deformation is calculated on each time step of the combined fluid/solid transient solution. As the finger sticks and pads deform under pressure, the flow zones' (Zones 1-4; Figs. 3 and 4) boundaries change shape correspondingly. Each resulting deformed shape is then used as initial geometry for the next time step. Calculations are performed until consecutive iterations converge to a final equilibrium shape. Typical computations performed on a Pentium 4 PC with $500 \mathrm{Mb}$ of RAM required a considerable amount of disk swapping. The time required to complete a typical calculation was approximately 15 central processing unit (CPU) hrs.

\section{Boundary Conditions}

In a typical seal operation there are high hydrostatic pressures in the front cavity of the seal (Zone 1) combined with a strong rotational flow in the vicinity of the shaft (Zone 2, Fig. 2). The low-pressure cavity behind the seal, Zone 3 , is at atmospheric pressure. The lower boundary of the computational domain is the shaft surface and has specified rotational boundary conditions. We used linear velocities of $100 \mathrm{~m} / \mathrm{sec}$ for the three-dimensional cases and $200 \mathrm{~m} / \mathrm{sec}$ for the two-dimensional cases. Outlet cavity conditions require a free-floating boundary to allow flow to develop as it may, in order to satisfy pressure and mass flow continuity conditions. For computational reasons, notwithstanding a certain computational time penalty, this boundary was moved farther downstream such that it would not affect the flow in the area adjacent to the second rows of fingers. Table 1 presents further characteristics of finger geometry, material, and grid specifications.

For the purpose of this study we utilized velocity-type boundary conditions at the inlet of Zone 1 . They are easier to implement and make for a more robust computational scheme while providing a higher convergence rate. On the top and bottom sides of computational domains we specified free-floating outlet conditions. We have specified symmetry boundary conditions at the top and bottom boundaries of the interstices (Fig. 4). Not only are these conditions possible due to the repetitive nature of the fingers and their contiguous interstices, but they are also of great benefit to the overall computational process by eliminating the need to simulate the entire circumference of the seal. Solid wall boundary conditions for the flow are applied at the
TABLE 1

Measurements and specifications

\begin{tabular}{ll}
\hline Diameter of shaft & 5.090 in $(129.3 \mathrm{~mm})$ \\
Inner diameter of the aft-finger pad & $5.091 \mathrm{in}(129.3 \mathrm{~mm})$ \\
Initial size of the pad gap (radial) & $0.0005 \mathrm{in}(0.0127 \mathrm{~mm})$ \\
Inner diameter of the forefinger & $5.120 \mathrm{in}(130.048 \mathrm{~mm})$ \\
$\begin{array}{l}\text { Gap of the forefinger (radial) } \\
\text { Axial width of the pad }\end{array}$ & $0.015 \mathrm{in}(0.381 \mathrm{~mm})$ \\
$\begin{array}{l}\text { Outer diameter of the finger } \\
\quad \text { root (stick) }\end{array}$ & $5.100 \mathrm{in}(2.54 \mathrm{~mm})$ \\
$\begin{array}{l}\text { Young modulus } \\
\text { Grid }\end{array}$ & $30 \mathrm{E}+6 \mathrm{psi}(207 \mathrm{GPa})$ \\
$\quad \begin{array}{l}\text { Total number of cells } \\
\quad \text { Total number of structured cells }\end{array}$ & 7561 \\
$\quad$ Unstructured cells & 8720 \\
\hline
\end{tabular}

stationary cavity walls and zero displacements for FEA at the finger-mounting locations.

\section{RESULTS AND DISCUSSION}

The results presented here are partitioned in two sections. The first refers to two-dimensional quantitative results that investigate flow, pressures, and pad deformations in the radial-axial plane. The second section examines the flow patterns around a pair of in-line fingers in a three-dimensional setup.

\section{Two-Dimensional Analysis}

In this "reduced" model, all the flow passes through a twodimensional axial cross-section (Fig. 2). No leakage flow is allowed between the neighboring fingers in a circumferential direction. Thus, it represents a limiting case for the finger seal. For all practical purposes the geometry of Figure 2 is equivalent to that of continuous compliant disks around the circumference. However, the stick and pad of the finger can move in both the axial and the radial directions. In this configuration the leakage flow is due only to the axial pad lift, which creates a radial gap clearance between the aft-finger pad and the runner. The forefinger is designed with a gap of 0.015 in $(0.381 \mathrm{~mm})$ above the shaft, thus not being able to seal at all. In the environment of an actual finger seal with staggered fingers, the role of this forefinger is to close (seal) the top and bottom gaps between adjacent fingers on a laminate, as well as eliminate Zone 4 (Fig. 2) when the high-pressure side presses the rows of fingers together. Thus, one can extrapolate and assume that a two-dimensional model will generate an "idealized" and lower limiting case. The two-dimensional approach considers an axisymmetrical model of rotational flow, with rotational speed specified on the shaft/runner surface. The fore- and aft-fingers deform under the pressure forces and change the clearance profile between the finger pad and the runner. That affects finger seal performance and its ability to lift and ride on the film surface. The Zone 1 inlet velocity boundary condition has been set 


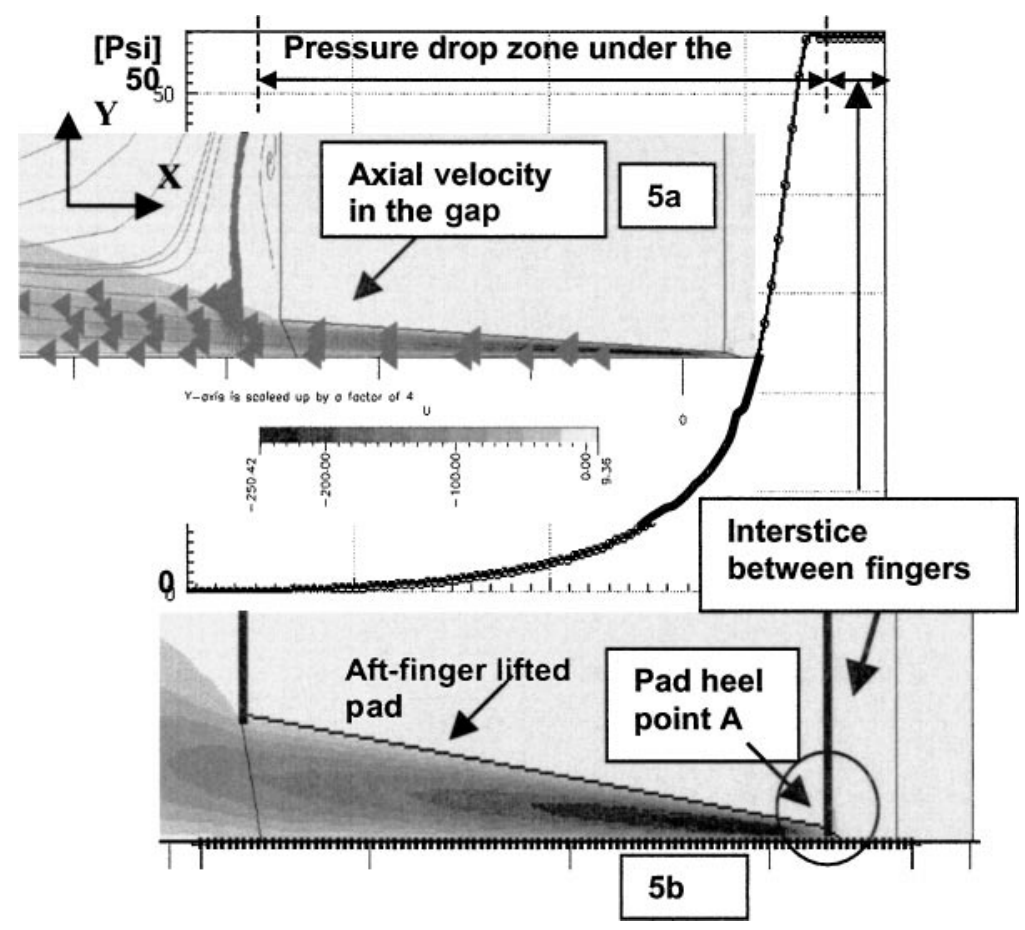

FIGURE 5

(a) Axial velocity profiles in the gap. (b) Pressure profiles under the pad and associated velocity contour profiles.

to $1.64 \mathrm{ft} / \mathrm{sec}(0.5 \mathrm{~m} / \mathrm{sec})$ and the shaft is rotating at $656 \mathrm{ft} / \mathrm{sec}$ $(200 \mathrm{~m} / \mathrm{sec})$.

Figure 5a (inset) presents the total velocity contours and, superimposed on them, the axial velocities as the flow progresses through the gap. One must note that even though inlet velocity boundary condition was very low, the maximum velocity in the gap reached $938.32 \mathrm{ft} / \mathrm{sec}(286 \mathrm{~m} / \mathrm{sec})$. The corresponding pressure drop was approximately $54.7 \mathrm{psi}(377 \mathrm{kPa})$ and the mass flow rate $7.1 \mathrm{E}-4 \mathrm{lbm} / \mathrm{sec}(3.22 \mathrm{E}-4 \mathrm{~kg} / \mathrm{sec})$. The pressure drop in the gap is shown in Figure 5b, which, for the reader's orientation, we have superimposed below the length of the gap containing the velocity contours. Note that each dot on the pressure curve corresponds to a dot on the abscissa of the superimposed gap. One can notice that the pressure in the high-pressure cavity does not drop at all as the flow enters the recessed $(0.015$ in $(0.381 \mathrm{~mm}))$ padless forefinger and crosses the interstice between the two fingers. However, the entrance under the aft-finger gap engendered a quick and steep pressure drop. As it appears from the further study of Figure $5 b$, the pressure under the pad became close to the low cavity pressure a little bit ahead of the trailing edge of the pad, indicating that possibly the pad can be shortened. Because the pad was positioned with a very small gap above the shaft, the axial flow under it was completely pressure-induced and the pressure generated both lifted and rotated the pad with respect to its heel point $\mathrm{A}(\mathrm{x}=\mathrm{y}=0)$. The effects of rotation were accounted for in the framework of an additional equation for rotational velocity in the axisymmetrical coordinates.
For the operational conditions with finger elastic deformation, pressure drops across the pad decreased from 54.7 psi $(377 \mathrm{kPa})$, with shaft rotation, to $13 \mathrm{psi}(90 \mathrm{kPa})$, without rotation. In the case of the nondeforming finger pressure drop decreases from $84 \mathrm{psi}(580 \mathrm{kPa})$ with shaft rotation to $72 \mathrm{psi}$ $(500 \mathrm{kPa})$ without rotation. Figures $6 \mathrm{a}$ and $6 \mathrm{~b}$ present the deformation of the pad under static pressure effects. The static pressure acting in Zone 1 causes an axial displacement and deformation of both the stick and the pad as shown in Figure 6a. Note the significant axial deformation of 0.004 in $(-1.1 \mathrm{E}-4 \mathrm{~m})$. Figure $6 \mathrm{~b}$ presents the radial lifting and deformation of the stick-pad assembly. A study of the scale bar reveals that the heel $\mathrm{A}$ is actually deflected downward toward the runner, causing a sort of a pinch point and reducing further the seal clearance. The trailing end of the pad deflects upward by approximately 0.0017 in $(4.2 \mathrm{E}-5 \mathrm{~m})$. It is worth noting that the forefinger stick deformations and motion, either radially or axially, is negligible since the axial momentum imparted by the flow (running at $1.64 \mathrm{ft} / \mathrm{sec}(0.5 \mathrm{~m} / \mathrm{sec}))$ is very low.

\section{Three-Dimensional Analysis}

Three specific cases were studied. In all cases the pair of fingers was arranged in line and the flow moves were unrestrained around the fingers through the in-line interstices. Such a situation is equivalent to the case in which the axially staggered finger laminates of a finger seal (Figs. 1b and 2) do not seal the interstices between the adjacent fingers distributed in the circumferential direction. 


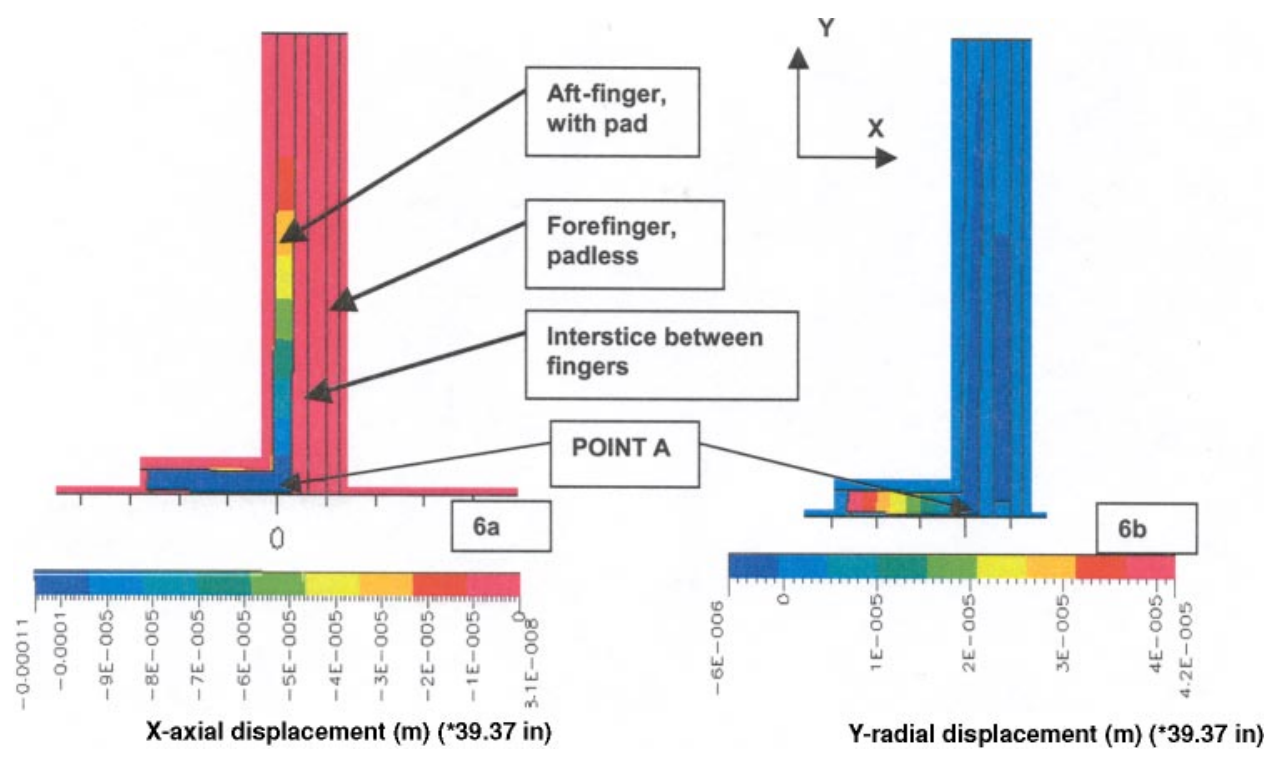

FIGURE 6

Axial and radial displacements of the pad.

\section{High Axial Velocity $\left(V_{\text {axial }}=328 \mathrm{ft} / \mathrm{sec}(100 \mathrm{~m} / \mathrm{sec})\right)$}

Figure 7 presents the numerical simulation of the flow across a pair of fingers like those shown schematically in Figure 2 . The high-pressure finger was padless while the low-pressure finger contained a pad, as shown in the figure. The three-dimensional rendition can be revisited in Figure 4. The fingers separated the high-pressure Zone 1 from the low-pressure Zone 3. As already mentioned, the upstream boundary conditions were velocity driven. Study of the figure indicates that the main path for flow leakage is through the bottom and top interstices, which represent gaps between sequential fingers. One can clearly see (as marked) particle tracer trajectories flowing through the top gap and the bottom gap, respectively. The axial flow effect is so strong in Zone 1 in the vicinity of the fingers that no flow trajectories are visible in the circumferential direction. However, in Zone 3, as the computation moves away from the finger area, there seem to appear circumferentially oriented flow patterns. This can be attributed to the decrease in the axial momentum of the flow as it passes through the top and bottom gaps.

\section{Medium Axial Velocity $\left(V_{\text {axial }}=164 \mathrm{ft} / \mathrm{sec}(50 \mathrm{~m} / \mathrm{sec})\right)$}

Figure 8 presents an intermediate case in which the axial velocity has been decreased by a factor of two. Figure 8 a presents the flow in Zone 1 in the vicinity of the pair of fingers. It can be seen that while far upstream the axial effects are undeterred, as the flow moves closer to the fingers, due to the necessary change in direction, the axial momentum diminishes considerably, and in the region of the pads the flow starts a strong circumferential motion. Figure $8 \mathrm{~b}$ presents the flow both in the low-pressure Zone 2 and in the interstice Zone 4 between the fingers. In both

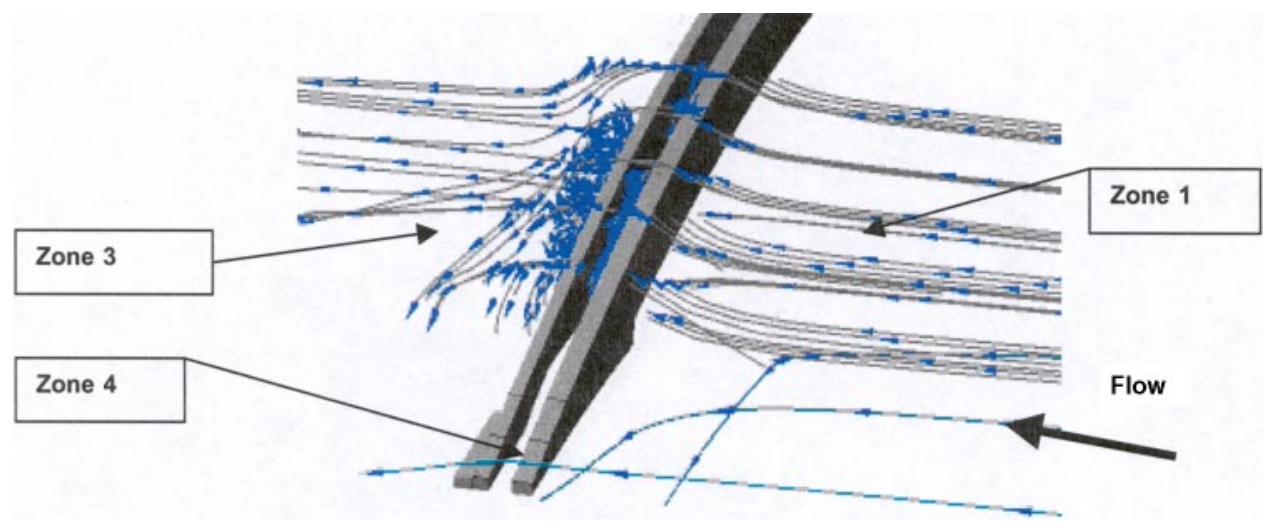

FIGURE 7

Flow/leakage between high-pressure Zone 1 and low-pressure Zone 3 when the shaft interface velocity is $100 \mathrm{~m} / \mathrm{sec}$ and the inlet axial velocity of flow to Zone 1 is $100 \mathrm{~m} / \mathrm{sec}$. 


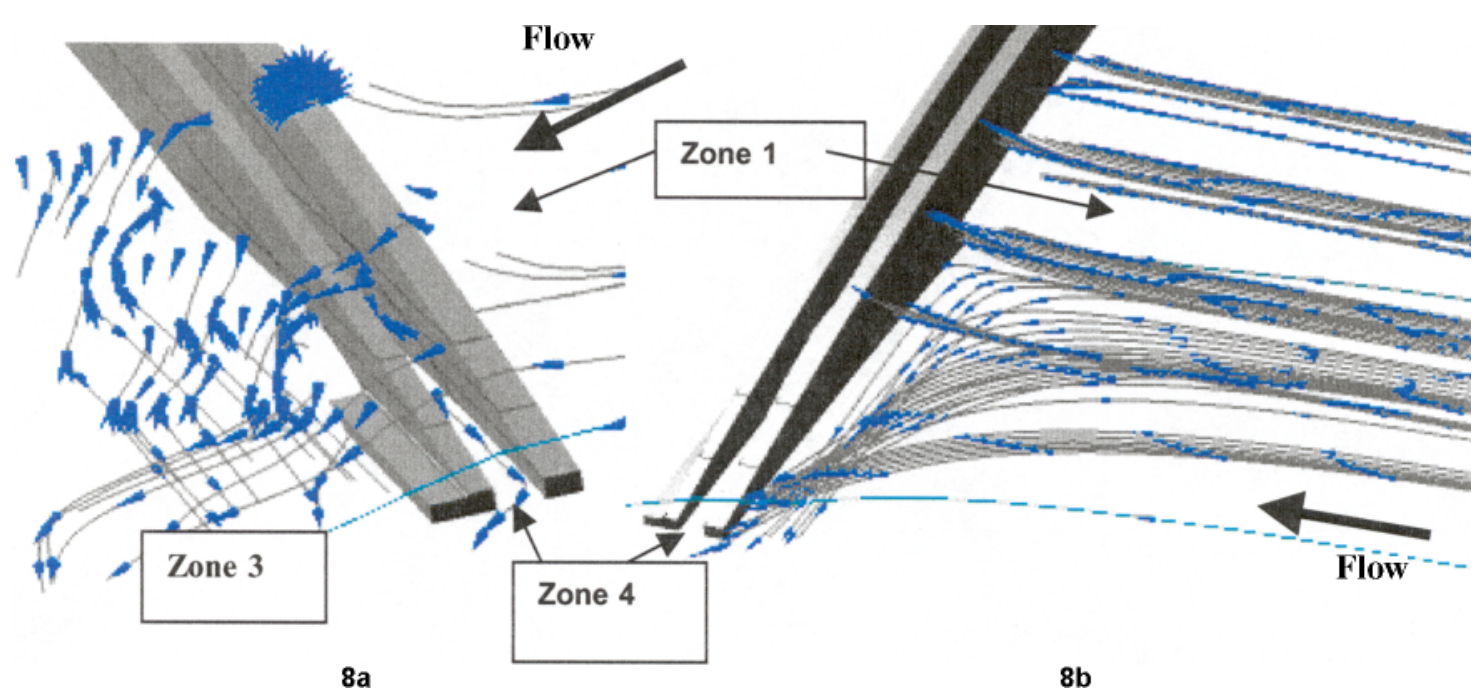

FIGURE 8

Flow/leakage between high-pressure Zone 1 and low-pressure Zone 3 when the shaft interface velocity is $100 \mathrm{~m} / \mathrm{sec}$ and the inlet axial velocity of flow to Zone 1 is $50 \mathrm{~m} / \mathrm{sec}$.

zones, the circumferential momentum is just starting to dominate, forcing the flow to strongly change direction and follow the motion of the shaft. However, one can also see clearly that the axial flow still plays a significant role. The apparition of the circumferential flow is a sign that the sealing effects are increasing because such circumferential flow itself acts as a barrier to leakage.

\section{Low Axial Velocity $\left(V_{\text {axial }}=3.28 \mathrm{ft} / \mathrm{sec}(1 \mathrm{~m} / \mathrm{sec})\right)$}

Figure 9 presents the flow around the two in-line fingers when the velocity boundary condition at the inlet of Zone 1 has been decreased by a factor of 100 from the high-speed original case presented. One can observe that the flow is now oriented predominantly in the circumferential direction in all three zones shown
$(1,4$, and 3$)$. The details in Figures $9 \mathrm{a}$ and $9 \mathrm{~b}$ show in three dimensional format the flow in the "in-between" finger interstice (Zone 4) as well as the regions immediately adjacent to the high-pressure finger (Zone 1) and low pressure finger (Zone 3).

\section{CONCLUSIONS}

The two- and three-dimensional numerical experiments considered here represent two limiting cases for the behavior of a finger seal. Thus, the two-dimensional case is equivalent to a finger seal in which there are no flow-through interstices between the fingers, whereas the three-dimensional cases present the worst-case scenario, that is, when the interstices between sequential fingers act as in-line sequential orifices with practically no capability of sealing. The results (See Figs. 5 and 6) show that

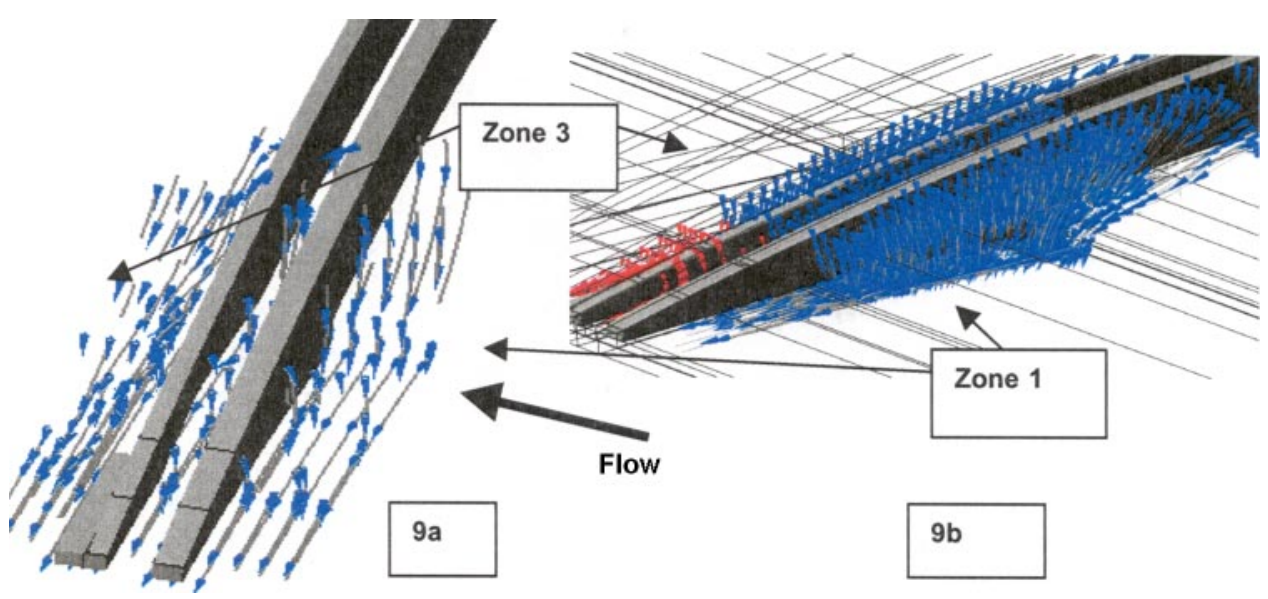

FIGURE 9

Flow/leakage between high-pressure Zone 1 and low-pressure Zone 3 when the shaft interface velocity is $100 \mathrm{~m} / \mathrm{sec}$ and the inlet axial velocity of flow to Zone 1 is $1 \mathrm{~m} / \mathrm{sec}$. 
the finger pad develops a deformation with rotation in the axial direction, becoming misaligned longitudinally with respect to the shaft. This imposes the necessity of designing the pads for minimum axial deformation and rotation. For the geometry of the pad studied here, it was found that in the three-dimensional case the radial motion of the pad upward was only minute. The three-dimensional studies of the flow showed that the velocity and geometry of the flow in Zone 1 (high pressure) influenced decisively the flow in the vicinity of the fingers. Thus, high axial velocity flows easily penetrated the zones inbetween sequential fingers, while low axial velocities allowed rotational effects in the vicinity of the fingers to take over. As well, it is clear that the width of the interstices between the fingers should be as small as possible in order to minimize leakage through the body of the finger seal. Finally, the study presented here provides insights into the physics of the compliant fingers and, in the authors' opinion, represents a first step toward the simulation of the staggered geometry of the finger seal.

\section{REFERENCES}

Arora, G. K. 1998. Noncontacting Finger Seal with Hydrodynamic Foot Portion. Patent No. 5,755,445.

Arora, G. K., Proctor, M. P., and Steinetz, B. M. Pressure-balanced, low-hysteresis finger seal test results. NASA Technical Memorandum 1999-209191.

Atkinson, E., and Bristol, B. 1992. Effects of material choices on brush seal performance. Lubrication Engineering 48:740-746.

Braun, M. J., Hendricks, R. C., and Canacci, V. 1990. Nonintrusive qualitative and quantitative flow characterization and bulk flow model for brush seals, 1611-1616. Proceedings of the Japan International Tribology Conference. Nagoya, Japan.

Chupp, R. E., and Dowler, C. A. 1991a. Performance characteristics of brush seals for limited life engines. Proceedings of the 36th ASME International Gas Turbine Conference. Paper No. 91-GT-281. Orlando, FL. J. of Engineering for Gas Turbines and Power, v. 115, p. 390, 1993.

Chupp, R. E., Holle, G. F., and Dowler, G. A. 1991b, June. Simple leakage flow model for brush seals. Proceedings of the AIAA/SAE/ASME 27th Joint Propulsion Conference, Paper No. 91-1913. Sacramento, CA.

Chupp, R. E., and Holle, G. F. 1994, June. Generalizing circular brush seal leakage through a randomly distributed bristle bed. Proceed- ings of the International Gas Turbine and Aeroengine Congress and Exposition, Paper No. 94-GT-71. The Hague, Netherlands.

Ferguson, J. G. 1988, June. Brushes as high-performance gas turbine seals. Proceedings of the ASME Gas Turbine and Aero Engine Congress, Paper No. 88-GT-182. Amsterdam, The Netherlands.

Forry, J. M. 1993. High-temperature brush seals and high-pressure ratio swept fan program. High Temperature Brush Seals, vol 1, Wright Labs. Technical Report No. WL-TR-94-2007. Dayton, Ohio: Wright Labs.

Hendricks, R. C., Schlumberger, S., Braun, M. J., Choy, F. K., and Mullen, R. 1991, June. A bulk flow model of a brush seal system. Proceedings of the 36th ASME International Gas Turbine Conference, Paper No. 91-GT-325. Orlando, FL.

Hendricks, R. C., Griffen, T. A., Bobula, G. A., Bill, R. C., and Howe, H. W. 1992. Integrity testing of brush seal in a T-700 engine, 117-138. NASA Lewis Research Center Seals Workshop, Publication 10124. Cleveland, OH: NASA.

Hendricks, R. C., O’Halloran, B., Arora, G., Addy, H. E., Flowers, J., Carlile, J., and Steinetz, B. M. 1994. Advances in contact sealing, 363-371. Advanced Earth-to-Orbit Propulsion Technology, NASA CP 3282 .

Heydrich, H. 1991. Bi-directional Finger Seal. Patent 5,031,922.

Johnson, M. C. 1992. Laminated Finger Seal with Logarithmic Curvature. Patent 5,108,116.

Lattime, S. B. 2001. A Hybrid Floating Brush Seal (HFBS) for improved sealing and Wear Performance in Turbomachinery Applications ( $\mathrm{PhD}$ diss, University of Akron, Ohio).

Ludwig, L. 1978. Gas path sealing in turbine engines. NASA Techinical Memorandum. NASA TM-73890, also advising group for aerospace research and development, AGARD, Seal Technology in Gas Turbine Engines, Paper No. 237. AGARD Publications, April 1998.

Mackay, C. G. 1991a. Laminated Finger Seal. Patent 5,042,823.

Mackay, C. G. 1991b. Laminated Finger Seal. Patent 5,071,138.

Mullen, R. L., Braun, M. J., and Hendricks, R. C. 1990, July. Numerical modeling of flows in simulated brush seal configuration. Proceedings of the AIAA/SAE/ASME/ASEE 26th Joint Propulsion Conference, Paper No. 90-2141. Orlando, FL.

Proctor, M. P., Walker, J. F., Perkins, H. D., Hoopes, J. F., and Williamson, G. S. 1996. Brush seals for cryogenic applications: performance, stage effects, and preliminary wear results in $\mathrm{LN}_{2}$ and $\mathrm{LH}_{2}$. NASA Technical Paper No. 3536.

Steinetz, B. M., Hendricks, R. C., and Munson, J. Advanced seal technology role in meeting next generation turbine engine goals. NASATM 1998-206961. 

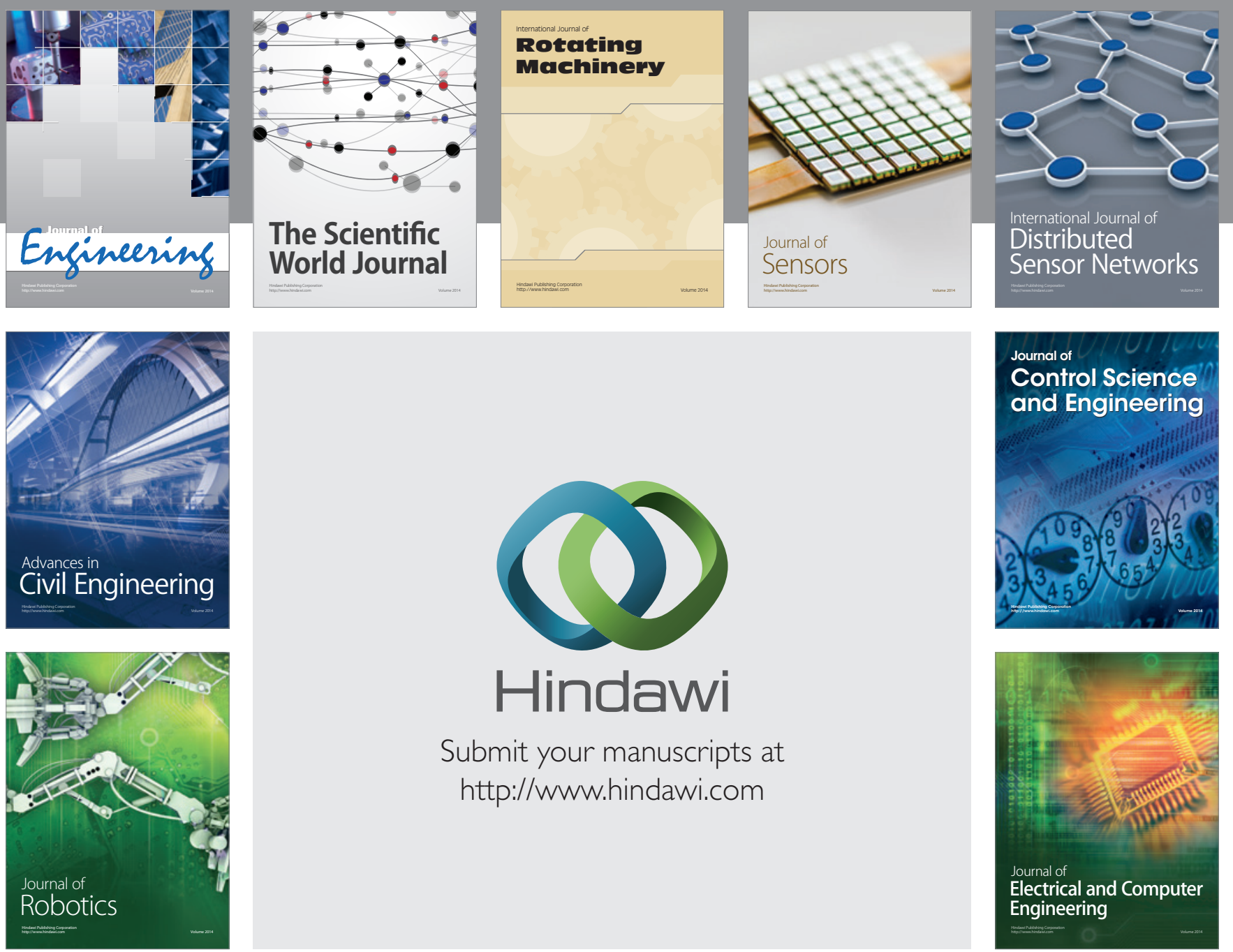

Submit your manuscripts at

http://www.hindawi.com
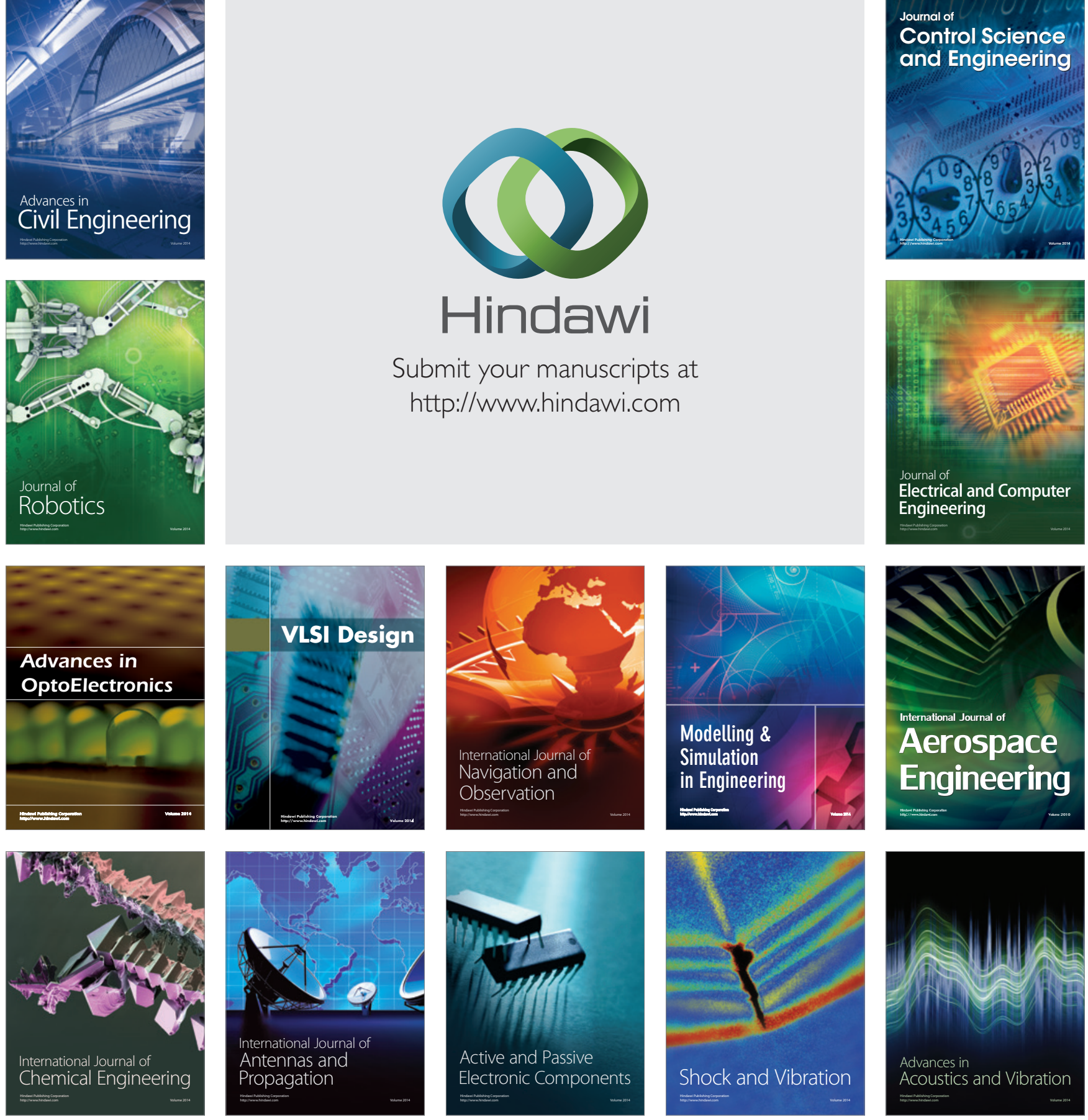